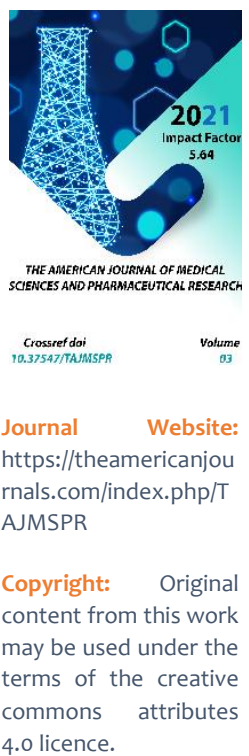

\section{Developing An Integrated Approach To Dental Implant Planning In Patients With Background Pathology And Covid -} 19

Javlonbek Jamoliddinovich Mannonov

Assistant, Department Of Operative Dentistry And Dental Implantology, Tashkent State Dental Institute, Tashkent, Uzbekistan

Barno Jurakhonovna Pulatova

Doctor Of Medical Sciences, Associate Professor Of Oral And Maxillofacial Surgery Department, Tashkent State Dental Institute, Tashkent, Uzbekistan

Mukhayo Ubayevna Asilova

Doctor Of The Highest Category, Chief Physician of The Research Institute Of Virology Of The Republican Specialized Center Of Epidemiology, Microbiology And Higher Practical Medicine, Tashkent, Uzbekistan

\title{
ABSTRACT
}

The use of intraosseous dental implants is one of the most distinctive features of modern dentistry. Implant-based prosthetic constructions are more physiological in comparison with traditional prosthetic techniques, since they transfer the chewing load directly to the alveolar bone of the jaw, besides, they do not require the preparation of neighboring teeth. Therefore, it is not surprising that in recent years, their use has become quite well known and appreciated by practitioners and patients alike. However, despite all the advantages, there are a number of contraindications and limiting factors to the use of implants $[5,11]$.

\section{KEYWORDS}

Background Pathology, Covid-19 Infection, Dental Implantation.

\section{INTRODUCTION}

At the present time scientists of the world pay increased attention to the problem of pandemic-Covid-19, all the population living on the Earth was exposed to coronavirus infection in mild and moderate form, $58 \%$ of the Earth population suffered from this infection in severe form, $27 \%$ with lethal outcome. Among the most common, it should be noted diseases of the cardiovascular system, namely hypertension and coronary heart disease, because it is in the presence of these nosological units, there is no consensus on the possibility of implantation. As the reasons for complications and unsatisfactory quality of treatment with the use of dental implantation experts consider in particular the insufficient professional training of specialists in the use of modern dental methods of diagnosis and 
treatment, unsatisfactory equipment of medical institutions with tools, devices and materials for treatment using modern minimally invasive technologies, as well as the lack of algorithms and standards for the use of such methods at various stages [3,5].

By now, dental implantology is a fundamentally sound approach to the treatment of adentia, which contributes to the clinical effectiveness of this complex of minimally invasive techniques and their widespread implementation in the practice of modern dentistry $[2,7]$. However, to date, reports on the results of comprehensive clinical and experimental studies in patients with a history of coronavirus infection and devoted to a comparative assessment of the clinical and economic effectiveness of microsurgical techniques for dental implantation in patients with partial and complete secondary adentia are practically absent in domestic dentistry $[1,9]$.

The implantation procedure in patients with somatic diseases, especially those of infectious and viral etiology, can itself provoke an exacerbation of the diseases that the patient is suffering from. Factors such as fear and pain can trigger myocardial ischemia, bronchospasm, hypertensive crisis, and somatic pathology can play a detrimental role in wound healing processes . It is known that the gingival papillae and periodontal pockets, where pathogenic flora accumulate, release endotoxins - into the bloodstream, which in turn, with the help of inflammatory mediators, cause endothelial damage. In cases (coronavirus infection, hypertension, diabetes mellitus, chronic obstructive bronchopulmonary disease) where this damage has already preceded, the inflammatory response may not be limited to the soft tissues of the periodontium, but may reach a systemic level with the development of infectious-toxic shock or sepsis $[4,6,8]$.

From an implantologist's point of view, it is important that long-term arterial hypertension, reduced immunity with Covid-19 and regular administration of hypotensive, anticoagulant drugs result in the phenomenon of "rarefaction" or "discharge", which is expressed as a reduction in the total surface area of the exchange vessels. To date, there are no criteria for extending the indications for implantation. The presence of concomitant somatic pathology in patients and coronavirus infection of viral etiology in need of dental implants has been the focus of attention of dentists, particularly in recent years, demonstrating the urgency of the problem on the one hand and its practical relevance on the other hand $[7,10,12]$.

Purpose of the study: To clinically substantiate the possibility of dental implantation in persons with coronavirus infection in preparation for prosthetics.

\section{MATERIALS AND METHODS}

To solve the goals and objectives of the study we conducted the examination and treatment of 77 patients who were operated on at the Department of Dental Surgery and Dental Implantology of the Tashkent State Institute of Dentistry for the period from 2018 to 2020 . The open method of dental implant management (with one-stage placement of gingival margin shapers) was used. A total of 53 men and 34 women were examined, 77 of whom had 191 intraosseous dental implants, DIO systems.

All patients were divided into three groups:

The first group consisted of patients with chronic coronary heart disease, in the form of 
stable angina pectoris, who had a moderately severe coronavirus infection. The second group - patients with essential hypertension without pathology COVID - 19 and the control group consisted of the practically healthy patients, the laboratory and functional examination of which revealed no general somatic pathology

Duration of postoperative follow-up

1 the day after the operation,

2 7-10 days after implant placing,

3 one month after implant placement,

4 after 3-6 months,

5. there after every 6 months from the beginning of the orthopedic structure. During the examination of patients at the preoperative stage, we used general clinical (anamnesis, examination of the intended implantation site, measurement of blood pressure and pulse), radiological (targeted radiography, panoramic zonography (orthopantomography) and computer tomography), functional and laboratory methods of examination (ultrasound Doppler flowmetry, ECG, periotestometry, blood chemistry)

Panoramic zonography (orthopantomography) in the course of our studies was performed with the use of different types of orthopantomographs We mainly used Phoenix (Finland, Radiante Ou) and PM 2002 CC (Finland, Planmeca Ou) devices. Imaging was performed at 60-70 kV, 5$10 \mathrm{~mA}$. Intra-oral radiography techniques were used in contact isometric imaging and parallel beam technique. In the present work, we used a CT scanner located in the Radiology and Radiology Department of the TMA 3 clinic,
"HiSpeed DX-I Plus" manufactured by General Electric with "Dento Scan" software.

To assess bone quality, we used the Misch and Judy, 1987 bone density classification compared with the X-ray tissue density scale expressed in Hounsfield units. In order to detect possible atherosclerotic lesions of the circulatory system and coagulation disorders, the following biochemical blood values were assessed during the planning stage of intraosseous dental implant surgery

1. Total cholesterol,

2. Low density lipoprotein cholesterol,

3. High density lipoprotein cholesterol,

4. Prothrombin according to Quick.

To confirm the diagnosis of Cavid-19, PCR studies and antibody testing for coronavirus infection were performed. SARS-CoV-2 IgMScreen(Covid-19), SARS-CoV-2 IgC Screen(Covid-19).

We used ultrasound Doppler flowmetry to assess the microcirculation in the soft and hard tissues at the proposed implant site. In cooperation with cardiologists and virologists, based on the results of studies and clinical experience, it was decided to perform intraosseous dental implantation only in patients with arterial hypertension of I and II severity degrees and with angina of functional classes I and II who had a history of coronavirus infection.

Statistical processing of the obtained data was performed using variational statistics, we calculated the arithmetic mean value, degrees of freedom of the arithmetic mean value The reliability of the differences between the mean values of the studied groups and the control groups was determined by Student's t-test. 


\section{RESULTS OF THE STUDY}

In cases with a history of arterial hypertension, there were no statistical differences between the study groups and the control group, only in the first degree of $\mathrm{AH}$. In cases with the second and third degree of severity, the process of bone remodelling was more strongly shifted towards bone resorption, as manifested by significant differences in bone volume when compared to the control group patients. A similar picture emerges when considering the dynamics of alveolar bone resorption in patients with CHD. Already in the angina II functional class group, a difference appears, although not yet statistically significant. In the presence of history of III and IV functional class angina, the difference is more pronounced and has a significant character, especially with COVID - 19. A qualitative analysis of bone tissue, expressed by measuring bone density by computed tomography, provided us with the following data:

In the lower jaw in hypertension of the first degree of severity, at the site of proposed implantation, the difference between the control group and the study group is present, although it is statistically insignificant. It is worth noting that the bone density in the premolars in degree I AH does not differ from that of the control group, while in degree II and III AH there is also a pronounced trend towards a lower density. In functional class I angina, comparing data on bone density in both the presumptive and premolar areas, there is no difference between the study and control groups. From the second class, there is no significant difference, while in the third and fourth classes there is a statistically significant difference that is directly proportional to the severity of the condition. Almost identical picture is formed by the analysis of the dynamics of changes in the density of the upper jaw depending on the type and severity of the accompanying cardiovascular pathology and the aggravation of the history of viral etiology. The only difference here is the physiological difference in bone density of the upper jaw compared to the lower jaw. The analysis of ultrasound Doppler flowmetry data reveals the following dynamics. Changes in the Gosling index, which reflects the degree of vascular elasticity, are insignificant in almost all study groups. Only in severe degree III arterial hypertension and functional class IV angina there is a statistically non-significant difference between the studied groups and the control group. This phenomenon can be explained by the fact that as a result of prolonged chronic stress on the vascular bed (high blood pressure, stenosis or occlusion of vessels by atherosclerotic plaques) blood is released through arteriolovenular shunts, i.e. a decrease of nutrient and increase of juxtacapillary blood flow. This is known to facilitate blood transport in the microcirculatory region, as the resistance of the bypass vessels due to their larger diameter and shorter length is much lower than that of the corresponding capillary networks.Therefore, changes in the Gosling index are not so pronounced. When assessing the indices of mean volumetric and mean linear velocities of blood flow in the area of prospective implantation, the influence of cardiovascular pathology on them becomes evident. Practically in all groups under study there is a statistically significant difference of the obtained data in comparison with analogous data in the control group. This observation allows speaking unequivocally about slowing of the microcirculatory blood flow in the region of the supposed implantation in the presence of previous history of $\mathrm{AH}$ and $\mathrm{CHD}$. 
It is also seen that there is a direct correlation between the severity of cardiovascular disease and changes of linear and volumetric blood flow velocities in patients with a history of viral infection. During biochemical blood investigation special attention was paid to lipid profile indexes (total cholesterol, low density lipoprotein cholesterol and high density lipoprotein cholesterol), because this group of tests is mostly used for diagnostics of atherosclerosis, which in its turn is an important pathophysiological link in development of cardiovascular diseases. In the presence of a history of stage I and II hypertension and functional class I and II angina pectoris, the values of the total blood cholesterol levels are usually within the physiological norm. For hypertension stage III and CHD stage III and IV, the values of total cholesterol were $6,24 \pm 1,23$ and $b, 72 \pm 1,31$, $7,62 \pm 1,68 \mathrm{mmol} /$ litre, the upper limit of the degrees of freedom of these indexes being above the physiological norm. This indicates the possibility of atherosclerotic vascular lesions of the circulatory system. The given conclusion is fully conforms to the statements of domestic and world literature that atherosclerotic lesions are probable but not obligatory concomitant risk factors of CVD (chronic coronary heart disease) and $\mathrm{AH}$ (arterial hypertension) development. In addition to the lipid profile, blood coagulation indices were also assessed, which is essential in any surgical procedure. In the biochemical analysis of the clotting system of hypertensive patients, all parameters are within the physiological norm. When comparing the values of the studied groups with those of the control groups, the level of statistical significance was greater than five per cent, i.e. these differences are statistically insignificant. All hypertensive patients showed a statistically significant increase in blood pressure before sedation. In the groups of patients with angina of functional class I. The heart rate, although clearly tachycardic, increased only slightly, especially in view of the psychological discomfort of the patients before the intervention.

The significant changes in the cardiovascular system values were noted almost immediately (3-5 min) after the sedative premedication with Relanium. Except for patients with $\mathrm{AH}$ of the second degree of severity, values of hemodynamic parameters came to the physiological norm limits in all groups. Although, the $\mathrm{AH}$ II group exceeded these limits, but the excess was insignificant and statistically non-significant. The indicated positive effect was stable throughout the surgical intervention and for some time after it.

\section{Post-operative monitoring}

As a rule, targeted radiographs in the area of the implants and standard orthopantomography were used to assess the tissue status of the bone-implant interface. 6 months after implantation and again at oneyear intervals, if the patient has no symptoms. 


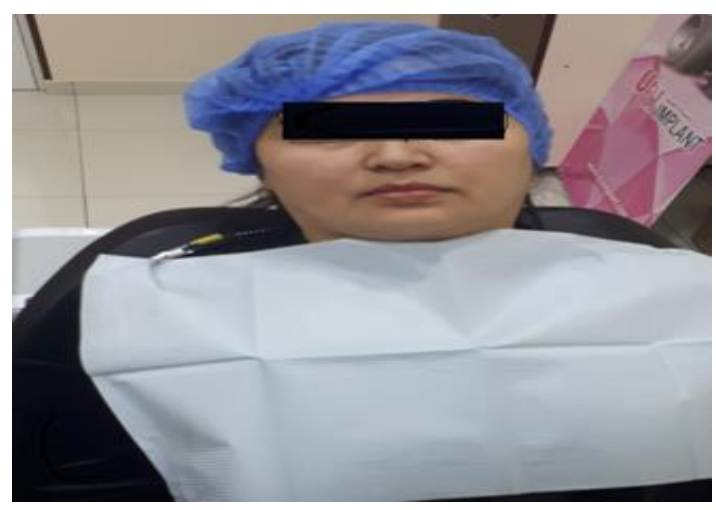

Fig.1. a-patient before surgery

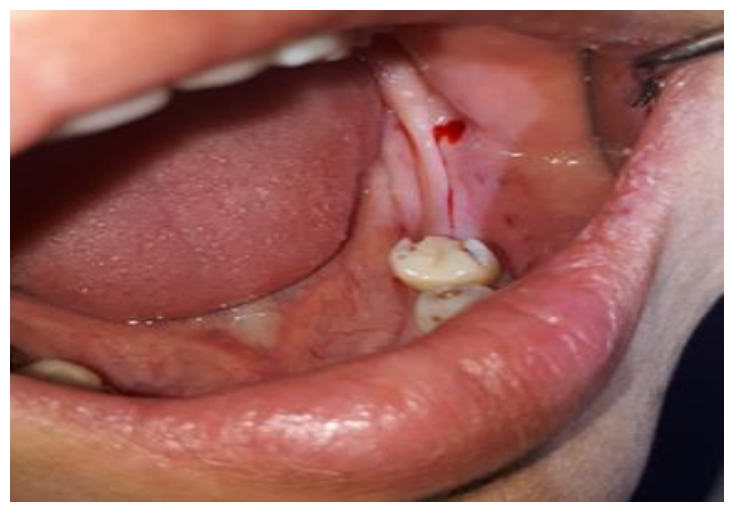

Fig.1. b- section of the oral mucosa;

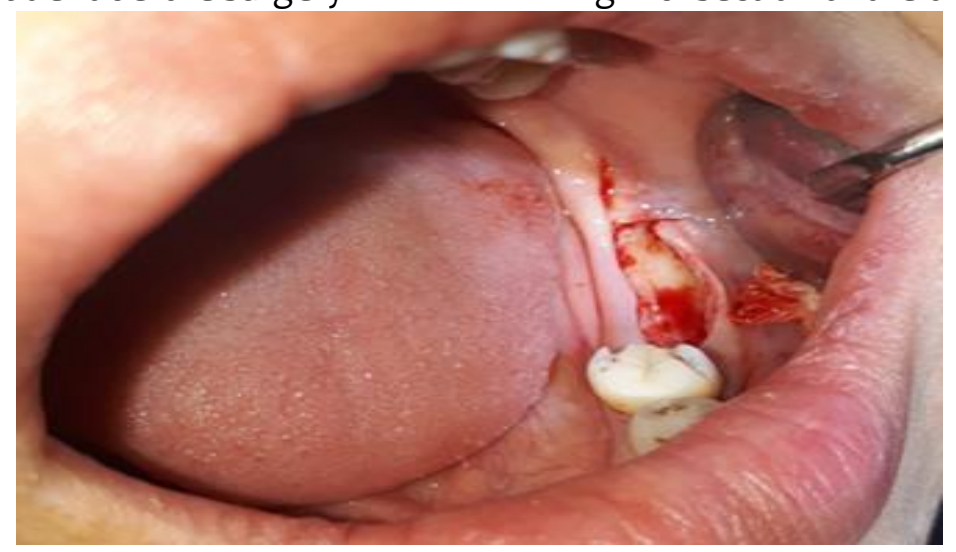

Fig.1. c. Patient in implant bed

One week after manipulation, patients in the control group usually show slight horizontal resorption in the area of the implant necks.

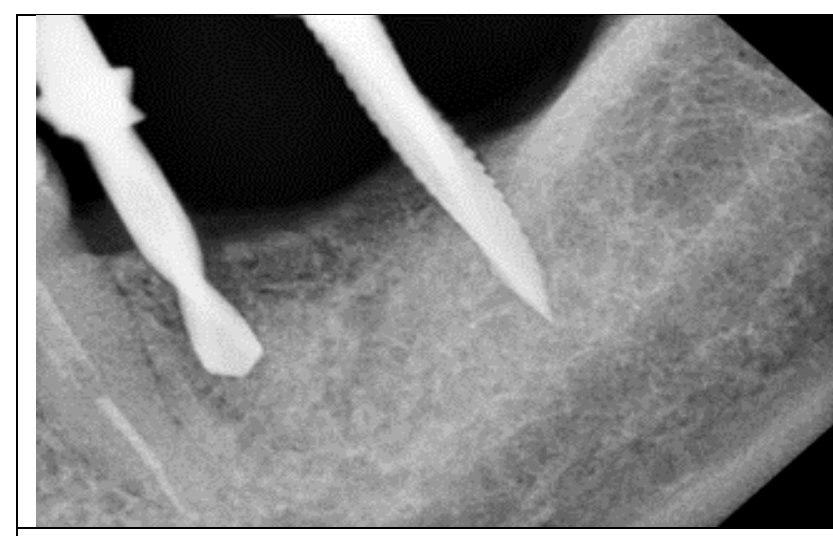

Fig.2 a- X-ray of the implant

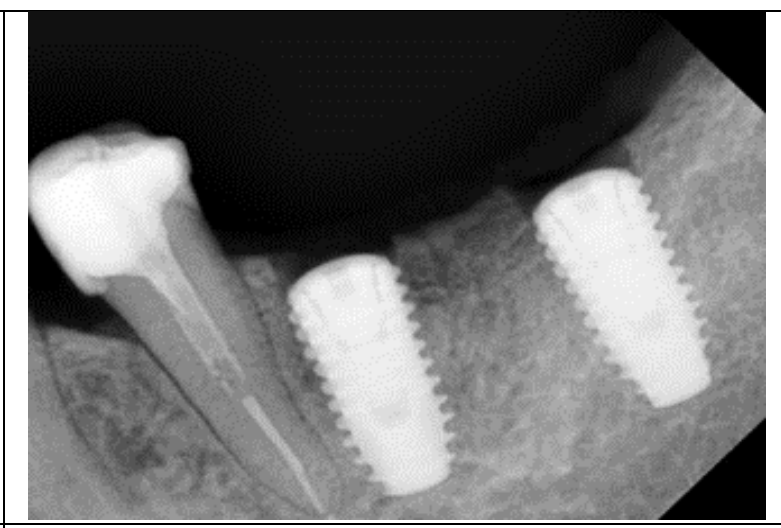

Fig.2 b X-ray of 2 implants placed 
This observation is described in the literature and can be explained by a shift in bone remodelling of the alveolar process towards resorption due to aseptic postoperative inflammation The average value of such resorption is approximately $0.5 \pm 0.1 \mathrm{~mm}$. There was a similar picture in the studied groups In the presence of arterial hypertension of the first and second stages, this index is approximately equal to $0.4 \pm 0.2 \mathrm{~mm}$ In angina of the first functional class, the resorption value was $0.5 \pm 0.2$, and in angina of the second functional class, $0.4 \pm 0.1$ It should be noted that these differences were not statistically significant, ie in all cases $\mathrm{P}<0.05$.

At the time of implant-supported prosthetic restorations, both horizontal and vertical bone resorption in the implant area was clinically negligible or absent in all groups observed.

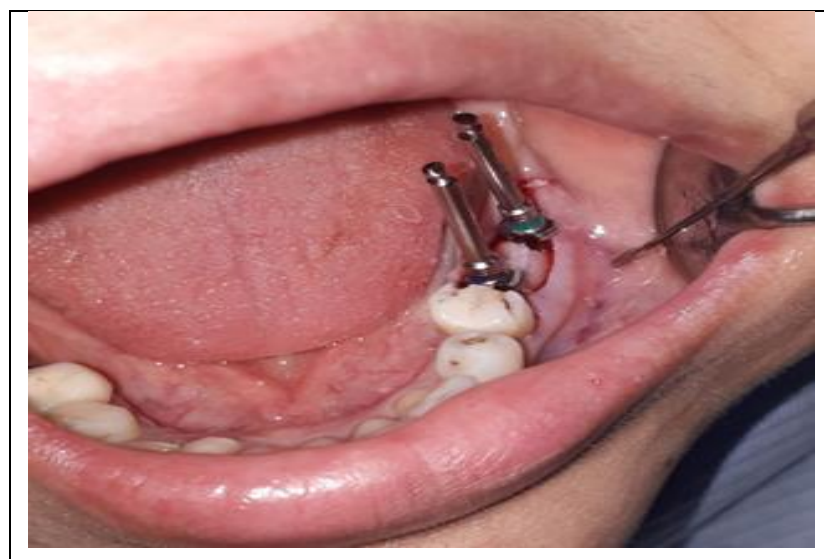

Fig.3 a-Checking the parallelism of inserted implants

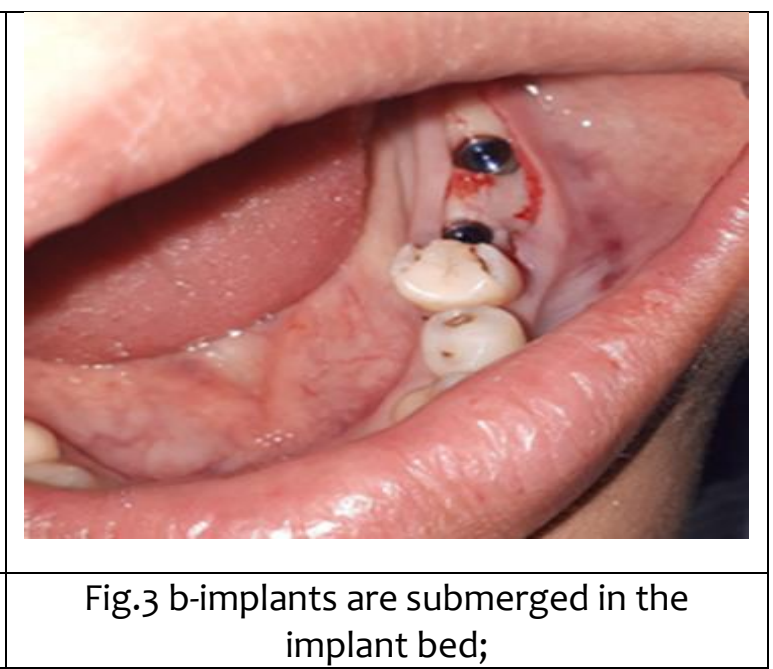

implant bed;

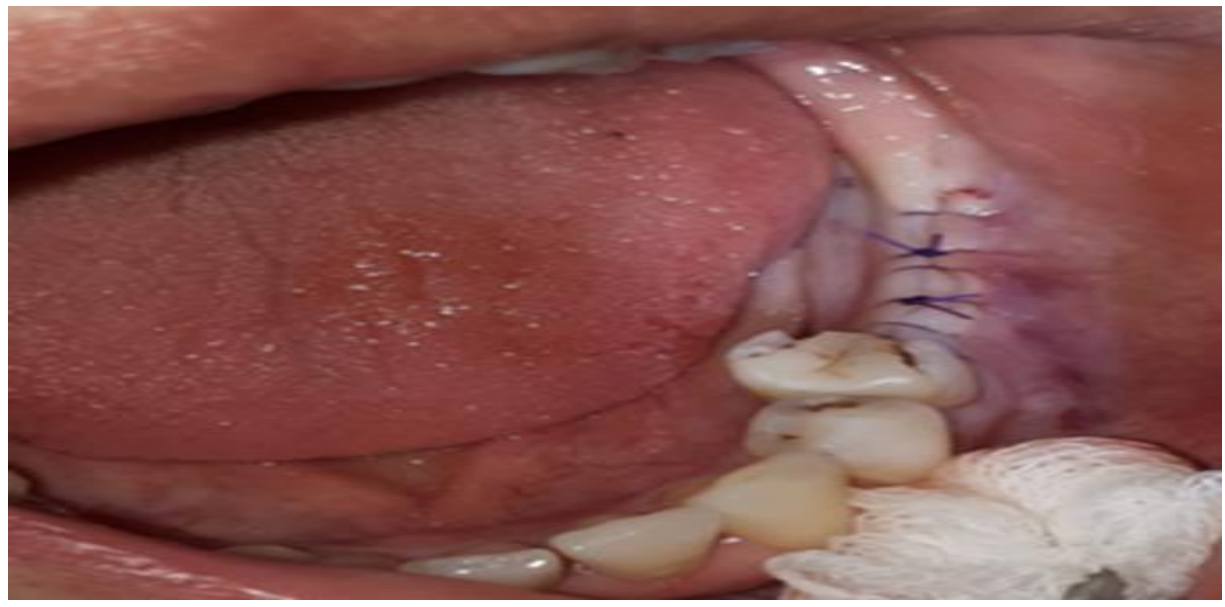

Fig.3 c-Sutures applied

Six months after the implants had become fully functional, radiographic examination showed no visible bone resorption in either the control or the study group. Two years after the surgical 
stage of dental implantation, a horizontal bone resorption in the bone-implant interface area was observed in some patients in the control group (40\%). There was also radiographically visible resorption in a proportion of patients (55\%) with arterial hypertension, with no apparent relation to the stage of the disease, with a mean value of $1.3 \pm 0.2 \mathrm{~mm}$. Horizontal resorption was also seen in $50 \%$ of patients in the CIBD group, with a mean value of $1.4 \pm 0.1$ $\mathrm{mm}$.

It should be noted that the presence of horizontal bone resorption of the boneimplant interface to a depth of no more than $1.5 \mathrm{~mm}$ at 2 years from the time of implant placement is normal and does not have any negative prognostic impact.

\section{CONCLUSIONS}

1. On the basis of the results of modern diagnostic methods the algorithm of actions of the medical personnel of the stomatological clinic allowing to achieve an objective estimation both of the general state of a patient with $\mathrm{AH}$ and $\mathrm{CICD}$ having Kavid-19 anamnesis and the local status of tissue in the area of the planned intervention was created for the first time.

2. By means of domestic and foreign literature sources analysis, numerous consultations with cardiologists and virologists on the basis of these researches there were worked out exact criteria of endosseous implantation realization possibility itself. According to these criteria, this procedure is possible only in cases of chronic coronary heart disease, functional class I and II angina pectoris, and grade I and II hypertension, even if there is a history of coronavirus infection, but only after a disease duration of 3 months.
3. In the planning of the implant surgery, there is evidence of a slowing of the microcirculatory blood flow in the tissues of the proposed intervention area, which undoubtedly has a negative effect on the osseointegration process.

4. The combined use of local anaesthesia with sedation premedication is necessary to achieve complete control of all components of the pain response.

\section{REFERENCES}

1. Kulakov A.A., Rabukhina N.A., Amkhadova M.A., Khamraev T.K., Petruhin P.V., Seiteev M.B., Duzhinsky S.V. (2003). The use of computer tomography in the planning of subperiosteal implantation. Collection of materials of scientific practical conference "Prospects of postgraduate education of stomatological profile experts Actual problems of stomatology" RMAGU, Moscow, p 290-292.

2. M.A. Amkhadova, A.A. Kulakov, S.V. Duzhinsky. (2004). Efficacy of use of subperiosteal implants at a significant atrophy of jaws. The Central Asian scientific and practical journal "Stomatologiya". №1-2(23-24). page 63-64.

3. Ismoilov, O. I., Murodkosimov, S. M., Kamalova, M. I., Turaev, A. Y., \& Mahmudova, S. K. (2021). The Spread Of SARS-Cov-2 Coronavirus In Uzbekistan And Current Response Measures. The American Journal of Medical Sciences and Pharmaceutical Research, 3(03), 45-50. 
4. Ilhomovna, K. M., Kadyrovich, K. N., \& Eryigitovich, I. S. (2021). Patient Demographics in Haemorrhagic and Ischaemic Stroke. Central Asian Journal of Medical and Natural Sciences, 2(4), 40-42.

5. M. I. Kamalova., N.K.Khaidarov., Sh.E.Islamov. (2020). Pathomorphological Features of hemorrhagic brain strokes, Journal of Biomedicine and Practice, Special issue, pp. 101-105.

6. Kadyrovich, K. N., Erkinovich, S. K., \& Ilhomovna, K. M. (2021). Microscopic Examination Of Postcapillary Cerebral Venues In Hemorrhagic Stroke. The American Journal of Medical Sciences and Pharmaceutical Research, 3(08), 69-73.

7. Shomurodov K.E. (2010). Peculiarities of cytokine balance in gingival fluid at odontogenicphlegmon of maxillofacial area. The doctor-aspirant. 42(5.1). pp.187-192.

8. Shomurodov, K. E. (2018). Comparative assessment of the influence of different methods of palatoplasty on the growth and development of the upper jaw in children with congenital cleft palate. European Science Review.-Vienna. Prague, (5-6), 7-11.

9. Isomov M.M., Shomurodov K.E. (2020). Peculiarities of rehabilitation of pregnant women with inflammatory diseases of maxillofacial area. International scientific-practical conference "Modern aspects of complex dental rehabilitation of patients with maxillofacial defects" May 21-22, Krasnodar. pp. 72-76.

10. The New York Times. Coronavirus Live Updates: W.H.O. Declares Pandemic as
Number of Infected Countries Grows. The New York Times. Available at https://www.nytimes.com/2020/03/11/ world/coronavirus-news.html\#link682e5bo6. March 11, 2020; Accessed: March 11, 2020

11. Khamdamov B.Z. (2020). Indicators of immunocitocine status in purulentnecrotic lesions of the lower extremities in patients with diabetes mellitus. American Journal of Medicine and Medical Sciences, 10 (7) 473-478 DOI: 10.5923/j.ajmm.2020. pp.1007.08.

12. Musaev U.Y.,Rizaev J.A., Shomurodov K.E. (2017). New views on the problem of dysemryogenesis stigmas of dentomandibular and facial system from the position of their formation in the disability of the population. Central Asian Scientific and Practical Journal "Stomatologiya "2017.-\#3-(68).-P.9-12 